\title{
Comparison of open reduction and internal fixation with total elbow arthroplasty for intra-articular distal humeral fractures in older age: a retrospective study
}

\author{
Jong Seok Baik, Sung Hyun Lee, Hyun Tak Kang, Tae Hyun Song, Jeong Woo Kim \\ Department of Orthopedic Surgery, Wonkwang University Hospital, Wonkwang University School of Medicine, Iksan, Korea
}

\begin{abstract}
Background: Intra-articular distal humeral fractures can be surgically challenging. It remains under discussion whether open reduction and internal fixation (ORIF) or total elbow arthroplasty (TEA) is more beneficial for treatment of the elderly. This study aimed to compare the clinical and functional outcomes of ORIF and TEA for managing intra-articular distal humerus fractures in patients aged 65 years or older. Methods: Patients who underwent ORIF $(n=28)$ or TEA $(n=43)$ for in intra-articular distal humerus fracture between May 2008 and December 2018 were reviewed. Range of motion, Mayo Elbow Performance Score (MEPS), Disabilities of the Arm, Shoulder, and Hand (DASH) score, radiologic outcomes, and surgical complications were evaluated at the final follow-up visit.

Results: The ORIF and TEA groups showed a mean arc of flexion-extension of $97^{\circ} \pm 21^{\circ}$ and $101^{\circ} \pm 12^{\circ}$, respectively. The mean MEPS and DASH scores were $94 \pm 15$ and $27 \pm 12$ points, respectively, in the ORIF group and $81 \pm 27$ and $47 \pm 28$ points in the TEA group. This difference was statistically significant. The incidence of total complications was similar between the groups.

Conclusions: In patients older than 65 years with intra-articular distal humerus fracture, ORIF had better outcomes than TEA.
\end{abstract}

Keywords: Fracture fixation; Total elbow replacement; Humeral fracture; Aged, 65 and over; Treatment outcome

\section{INTRODUCTION}

Distal humeral fractures account for $2 \%$ of all fractures in adults [1]. In younger patients, these fractures are mainly caused by high-energy trauma, whereas, among older patients, they are more often the result of a direct injury that occurs from falling. Anatomical joint reconstruction and stable fixation of intra-articular and comminuted fractures remain difficult to achieve, and the operation for displaced intra-articular distal humeral fractures is partic- ularly challenging.

Open reduction and internal fixation (ORIF) with double-locking plate osteosynthesis has become the gold standard for fixation of intra-articular distal humeral fractures, though the optimal positioning and configuration of the plates remain controversial [2-4]. In addition, elbow stiffness, malunion, nonunion, fixation failure, and ulnar neuropathy are common sequelae, with an overall complication rate greater than 35\% [5-9]. In older individuals with low bone densities, there is an increased risk of osteosynthesis failure [10].

Received: January 31, $2020 \quad$ Revised: March 22, $2020 \quad$ Accepted: March 22, 2020

Correspondence to: Jeong Woo Kim

Department of Orthopedic Surgery, Wonkwang University School of Medicine, 460 Iksan-daero, Iksan 54538, Korea

Tel: +82-63-859-1360, Fax: +82-63-852-9329, E-mail: serina@wonkwang.ac.kr, ORCID: https://orcid.org/0000-0002-0828-7179

IRB approval: Wonkwang University Hospital (No. WKUHIRB 2018-01-011)

Financial support: None.

Conflict of interest: None. 
Total elbow arthroplasty (TEA) is considered effective for treating nonunion, malunion, posttraumatic arthritis, and posttraumatic instability, with the literature reporting good to excellent results in $85 \%$ to $90 \%$ of patients at 5 to 10 years after surgery [11-13]. However, TEA for these injuries is technically difficult, and the complication rates are higher than those for elbows that have not undergone prior operations $[7,11]$. The purpose of this study was to compare the clinical and functional outcomes of ORIF with those of TEA for displaced intra-articular distal humeral fractures in patients aged 65 years and older.

\section{METHODS}

This study was approved by Institutional Review Board of Wonkwang University Hospital (IRB No. WKUHIRB 2018-01-011), and it was exempted from informed consent. We retrospectively reviewed the medical records of patients who underwent ORIF or TEA for intra-articular distal humeral fractures between May 2008 and December 2018. Eligible patients were those that met the following inclusion criteria: (1) aged of 65 years or older; (2) displaced and comminuted intra-articular distal humeral fractures requiring surgical treatment (AO/Orthopaedic Trauma Association [OTA] classification type 13C) [14], (3) surgical treatment with either ORIF with double-locking plate osteosynthesis or TEA, and (4) a minimum follow-up of 2 years.

Patients with extra- or partial-articular distal humeral fractures (AO/OTA classification types $13 \mathrm{~A}$ and 13B), intra-articular distal humeral fractures not requiring surgical treatment (AO/OTA classification type 13C), open fractures, vascular injuries, previous ipsilateral distal humeral fractures, pathologic fractures, or fractures with diaphyseal extension $\geq 8 \mathrm{~cm}$ were excluded. A total of $77 \mathrm{pa}-$ tients met the inclusion criteria of this study. Six patients died from unrelated causes. Of the remaining 71 patients, 28 underwent ORIF with double-locking plate osteosynthesis, while 43 underwent TEA. Patient demographics are summarized in Table 1.

Patient medical records were reviewed for demographic and perioperative data. All patients were invited for a clinical follow-up examination comprising evaluation of range of motion, elbow stability, and neurologic deficits. Additionally, pain status, patient satisfaction, and revision surgeries were documented. Finally, the Mayo Elbow Performance Score (MEPS) and Disabilities of the Arm, Shoulder, and Hand (DASH) score were examined. The functional outcome, based on the arc of motion, MEPS, and DASH score, as well as minor and major complications were evaluated and compared between the TEA and ORIF groups. All follow-up assessments were conducted by the same surgeon who performed the surgery.

\section{Surgical Techniques}

All surgeries were performed by the same senior surgeon (JWK).

\section{ORIF Group}

ORIF was performed using a posterior approach. After exposing the triceps muscle, the elbow joint was revealed by chevron osteotomy of the olecranon for accurate anatomical reduction. After temporary K-wire fixation of the fracture fragments, double-locking plates were applied to the medial and lateral sides of the distal humerus. The ulnar nerve was transposed into an anterior subcutaneous position at the conclusion of all surgical procedures. After postoperative immobilization for two days, passive range-of-motion exercise was performed for 6 weeks.

\section{TEA Group}

A Coonrad-Morrey semiconstrained prosthesis (Zimmer Biomet, Warsaw, IN, USA) was used in all cases. A midline triceps split or

Table 1. Patient demographics

\begin{tabular}{lccc}
\hline Variable & ORIF & TEA & P-value \\
\hline Sex $($ male:female) & $7(25): 21(75)$ & $9(21): 34(79)$ & 0.59 \\
Age $(\mathrm{yr})$ & $76(65-89)$ & $79(65-91)$ & 0.16 \\
BMI $\left(\mathrm{kg} / \mathrm{m}^{2}\right)$ & $24 \pm 3(19-30)$ & $23 \pm 4(15-34)$ & 0.49 \\
DM & $4(14)$ & $8(18.6)$ & 0.34 \\
Smoking & $2(7)$ & $2(4.6)$ & 0.28 \\
Follow-up period (mo) & $31(9-58)$ & $34(6-116)$ & 0.59 \\
AO classification & & & 0.31 \\
C1 & 8 & 10 & 10 \\
C2 & 2 & 23 & \\
C3 & 18 & & \\
\hline
\end{tabular}

Values are presented as number (\%), mean (range), or mean \pm standard deviation (range).

ORIF: open reduction and internal fixation, TEA: total elbow arthroplasty; BMI: body mass index; DM: diabetes mellitus. 
triceps-sparing approach was used. With the triceps-sparing approach, the surgeon used the working space created by condylar resection to perform TEA without detaching the triceps from the olecranon $[11,15]$. The ulnar nerve was transposed into an anterior subcutaneous position at the conclusion of all surgical procedures. After postoperative immobilization for 2 days, early functional mobilization was started without weight-bearing for 6 weeks.

\section{Statistical Analysis}

Statistical analyses were performed using IBM SPSS ver. 22.0 (IBM Corp., Armonk, NY, USA). The functional outcomes were statistically compared using the Mann-Whitney U-test as a two-way analysis of variance for independent factors.

\section{RESULTS}

\section{Clinical Outcomes}

In the ORIF group, the mean pain score was 3.1 (range, 1-6), mean MEPS was 94 (range, 75-100), and mean DASH score was 27 (range, 10-45). Based on MEPS, there were 20 excellent and seven good results. With respect to motion, average extension was $17^{\circ}$ (range, $5^{\circ}-40^{\circ}$ ), average flexion was $120^{\circ}$ (range, $80^{\circ}-140^{\circ}$ ), and average arc of flexion-extension was $97^{\circ}$ (range, $70^{\circ}-130^{\circ}$ ). Mean supination angle was $84^{\circ}$ (range, $80^{\circ}-90^{\circ}$ ), and mean pronation angle was $88^{\circ}$ (range, $75^{\circ}-90^{\circ}$ ) (Table 2 ).

All radiographs of fractures that healed without revision (all patients) showed anatomical reduction with stable fixation (Fig. 1). We did not intentionally rule out fractured patients who needed revision, but there was no patient who required it.

Table 2. Functional results comparing ORIF and TEA

\begin{tabular}{|c|c|c|c|}
\hline Variable & ORIF & TEA & P-value \\
\hline Pain (NRS) & $3.1(1-6)$ & $3.5(0-8)$ & 0.21 \\
\hline MEPS & $94 \pm 15(75-100)$ & $81 \pm 27(50-85)$ & $0.028^{*}$ \\
\hline DASH score & $27 \pm 12(10-45)$ & $47 \pm 28(10-75)$ & $0.038^{\star}$ \\
\hline \multicolumn{4}{|l|}{$\mathrm{ROM}\left(^{\circ}\right)$} \\
\hline Extension & $17 \pm 21(5-40)$ & $15 \pm 3(0-30)$ & 0.104 \\
\hline Flexion & $120 \pm 18(100-140)$ & $124 \pm 15(80-150)$ & 0.265 \\
\hline Flexion-extension & $97 \pm 21(70-130)$ & $101 \pm 12(80-140)$ & 0.089 \\
\hline Supination & $84 \pm 6(80-90)$ & $84 \pm 6(80-90)$ & 0.126 \\
\hline Pronation & $88 \pm 5(75-90)$ & $89 \pm 6(80-90)$ & 0.072 \\
\hline Satisfaction & & & - \\
\hline Excellent & $20(71.4)$ & $5(11.6)$ & \\
\hline Good & $7(25)$ & $18(41.8)$ & \\
\hline Fair & $1(3.5)$ & $14(32.6)$ & \\
\hline Poor & 0 & $6(14.0)$ & \\
\hline
\end{tabular}

Values are presented as mean (range), mean \pm standard deviation (range), or number (\%).

ORIF: open reduction and internal fixation, TEA: total elbow arthroplasty, NRS: numeric rating scale, MEPS: Mayo Elbow Performance Score,

DASH: Disabilities of the Arm, Shoulder, and Hand, ROM: range of motion.

${ }^{\star}$ Statistically significant.
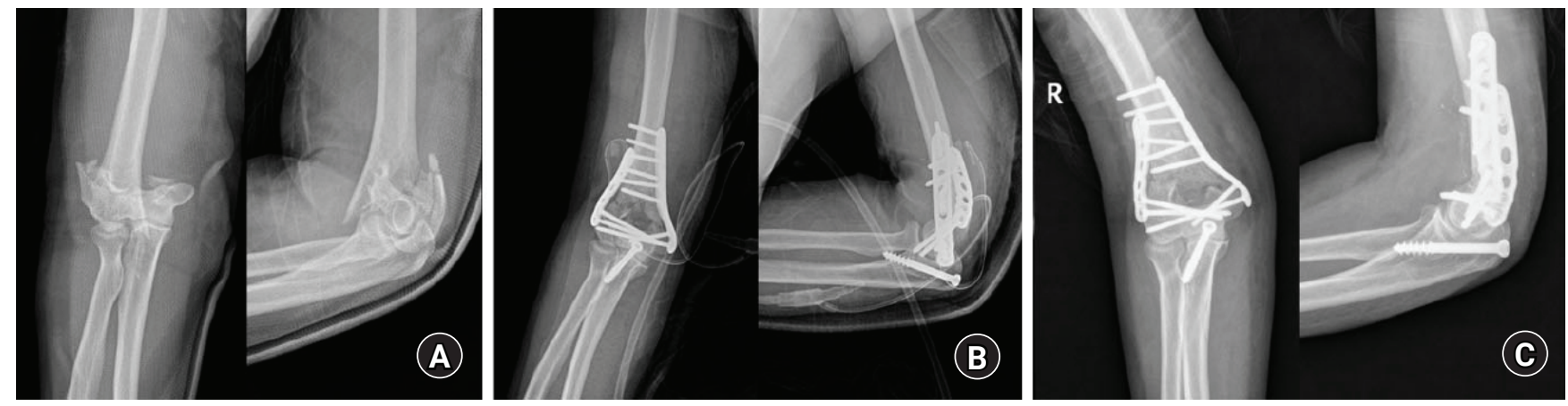

Fig. 1. Serial X-rays of open reduction and internal fixation (ORIF) case. (A) Anteroposterior and lateral X-rays of a 66-year-old female who suffered an $\mathrm{AO}$ classification 13C distal humerus fracture with a fall. (B) An X-ray performed after ORIF, showing dual-plate fixation. (C) Bone union after 6 months. 
Patients with infection were treated with antibiotics and did not need additional surgery. One patient had Brooker type III heterotopic ossification in the brachialis musculature.

In the TEA group, mean pain score was 3.5 (range, $0-8$ ), mean MEPS was 81 (range, 50-85), and mean DASH score was 47 (range, 10-75). Based on MEPS, there were five excellent and 18 good results. With respect to motion, average extension was $15^{\circ}$ (range, $0^{\circ}-30^{\circ}$ ), average flexion was $124^{\circ}$ (range, $80^{\circ}-150^{\circ}$ ), and average arc of flexion-extension was $101^{\circ}$ (range, $80^{\circ}-140^{\circ}$ ). Mean supination angle was $84^{\circ}$ (range, $80^{\circ}-90^{\circ}$ ), and mean pronation angle was $89^{\circ}$ (range, $80^{\circ}-90^{\circ}$ ) (Table 2 ).

At the final follow-up, incomplete radiolucent lines were seen around the humeral implant in six cases and in the ulnar implant in five cases. Wear of the polyethylene bushings at the hinge was directly correlated with follow-up duration. When we calculated satisfaction, the ORIF group (96.4\%) contained a higher percentage of patients who answered good or excellent compared with the TEA group (53.4\%).

\section{Complications}

Complications occurred in 13 patients in the ORIF group and 14 patients in the TEA group (Table 3). Two patients underwent revision in the TEA group, including one due to deep infection and one due to periprosthetic fracture with aseptic loosening of the humeral stem. These two cases in the TEA group were separated from the other, nonrevised TEA cases when measuring the clinical scores. The single case of deep infection occurred seven years after the initial TEA in a female patient. She underwent implant removal, insertion of antibiotic beads, and an installation of new prosthesis with antibiotic-loaded acrylic cement in the first-stage surgery. Six weeks after the first-stage surgery, revision TEA was performed after confirming no infection. Separately, two cases of superficial infection occurred in the ORIF group and were treated with intra-

Table 3. Complications

\begin{tabular}{lccc}
\hline Variable & ORIF & TEA & P-value \\
\hline Wound dehiscence & 0 & $1(2.3)$ & \\
Aseptic loosening & $1(3.5)$ & $2(4.7)$ & \\
Fracture & 0 & $1(2.3)$ & \\
Heterotrophic ossification & $1(3.5)$ & $7(16.3)$ & \\
Ulnar nerve symptom & $7(25)$ & $2(4.7)$ & \\
Infection & $2(7.1)$ & $1(2.3)$ & \\
Elbow stiffness & $2(7.1)$ & 0 & \\
Total & $13(46)$ & $14(32)$ & 0.18 \\
\hline
\end{tabular}

Values are presented as number (\%).

ORIF: open reduction and internal fixation, TEA: total elbow arthroplasty.

${ }^{\star}$ Flexion $<120^{\circ}$ and loss of extension $>30^{\circ}$. venous antibiotics without surgery.

In the TEA group, the case of periprosthetic fracture occurred around the tip of the humeral implant, which had completely loosened. Here, the humeral implant was replaced with a longer one and augmented using a humerus shaft strut allograft (Fig. 2). Another case of loosening occurred between the cement and both the humerus and the ulna, while screw-loosening occurred in one case in the ORIF group. Also, neurological complications occurred in nine cases, including two in the TEA group and seven in the ORIF group. These patients reported only dysesthesia of the fourth and fifth fingers without sensory or motor deficits, with all recovering. Heterotopic ossification occurred in eight patients, but there was no instability or other clinical symptoms, and no surgical treatment was required.

We classified aseptic loosening, fractures, and infection as major complications; thus, the ORIF group had mainly minor complications such as ulnar nerve symptoms. When we calculated the rate of major complications of total complications, three of 13 (23.1\%) in the ORIF group and four of 14 (28.6\%) in the TEA group had major complications. Thus, the rate was higher in the TEA group, although there was no statistical significance.

\section{DISCUSSION}

The purpose of this study was to compare the clinical and func-
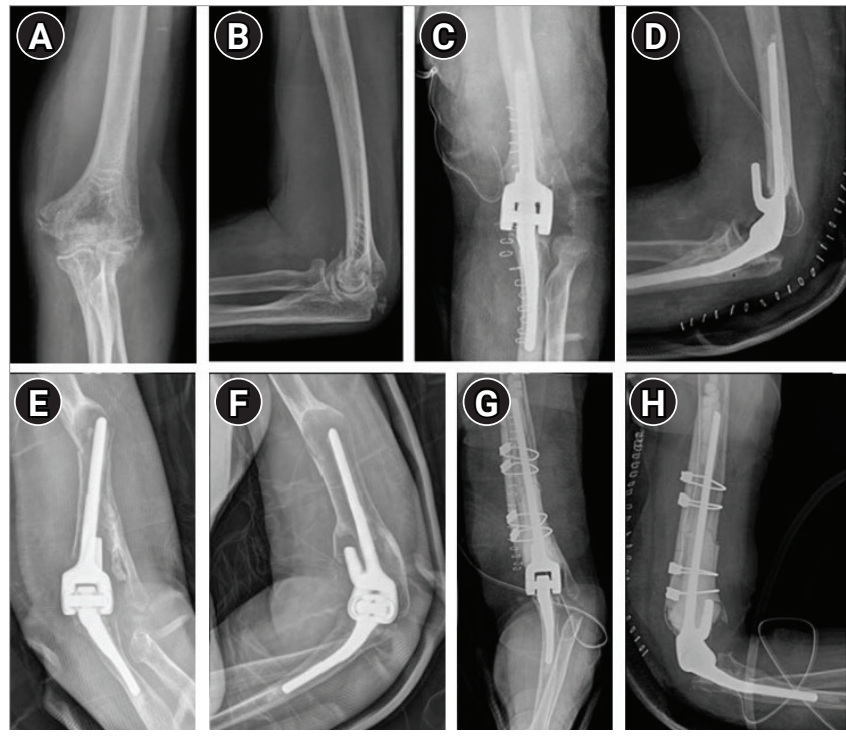

Fig. 2. A 75-year-old female suffered AO classification 13C distal humerus fracture with a slip down $(\mathrm{A}, \mathrm{B})$ and was treated with total elbow arthroplasty (TEA) (C, D). After 3 years, the patient complained of upper arm pain after carrying a heavy load. Periprosthetic fracture with aseptic loosening around the humeral stem was observed (E, F). Revision TEA with longer humeral stem and humerus shaft strut allograft was performed $(\mathrm{G}, \mathrm{H})$. The final functional outcome was relatively good. 
tional outcomes of ORIF with those of TEA for displaced intra-articular distal humerus fractures in patients aged 65 years or older. At the time of final follow-up, the ORIF group had significantly higher MEPS and DASH scores compared with the TEA group.

Although the recommended treatment for comminuted and displaced intra-articular distal humerus fractures is osteosynthesis, this method can be technically difficult, especially in older patients $[16,17]$. Previous data on ORIF for distal humerus fractures indicate that complications such as fixation failure, persistent pain and/ or stiffness, heterotopic ossification, ulnar nerve entrapment, nonunion, malunion, and posttraumatic arthritis are common in all age groups.

Several studies have suggested that primary TEA is a reliable treatment for severe intra-articular distal humerus fractures in elderly patients. Morrey et al. [18] reported a series of 21 patients with a mean age of 72 years who underwent primary TEA for comminuted distal humerus fractures. They reported good or excellent results in $95 \%$ of the patients at a mean follow-up of 3.3 years, with a reoperation rate of 5\% (one elbow). Gambirasio et al. [19] reported 10 women (mean age, 85 years) who underwent primary TEA. Of them, eight had an excellent outcome and two had a good outcome based on MEPS. Garcia et al. [20] evaluated 16 patients with a mean age of 73 years (range, 61-95 years) who underwent primary TEA with a mean follow-up of 3 years (range, $1.5-5$ years). These authors reported mean DASH score of 23 (range, 1-63) and mean MEPS of 93 (range, 80-100). Frankle et al. [21] reported 11 excellent, one good, zero fair, and zero poor results in patients who underwent TEA.

There are several studies comparing ORIF and TEA for comminuted intra-articular distal humerus fractures. Frankle et al. [21] reported better functional outcomes and higher MEPS in the TEA group as well as a higher incidence of complications in the ORIF group. In a prospective multicenter study, McKee et al. [22] reported improvement in MEPS and DASH scores in the TEA group relative to in the ORIF group. Egol et al. [23] reported no significant differences between the groups in a study comparing TEA and locking-plate osteosynthesis. Obremskey et al. [24] reported no strong evidence to choose one of two treatments for deployment in patients in their 60s who have a long predicted remaining lifespan and no comorbidities.

Ulnar neuropathy is a common complication after surgery. McKee et al. [22] reported that postoperative ulnar neuropathy was the single most common complication in their study comparing TEA and ORIF for distal humerus fractures. In our study, ulnar neuropathy was also the most common complication (nine cases) and was significantly more frequently observed in the ORIF group. We note that these complications occurred despite routinely trans- posing the ulnar nerve in all patients. Thus, the surgeon should be extremely cautious of the ulnar nerve during surgery. Rates of major complications, such as infection and loosening, were similar in the two groups.

Infection seems to occur due to swelling; soft tissue damage after trauma; or shear force during early range of motion or due to the large volume of the dual plate, which can irritate the skin because the elbow joint has thin soft tissue. In our study, the infection rate of the ORIF group (7.1\%) was not as high as reported in other researches [25]. Proper soft tissue control during the operation should be ensured.

Limitations of our study, including its retrospective design and the small number of patients, are comparable to those of previous studies but might have underpowered our study. Furthermore, despite a mean follow-up length of 34 months, it is difficult to compare the preoperative status between groups because only the final follow-up results were evaluated.

In older patients with intra-articular distal humeral fractures, those undergoing ORIF had better clinical outcomes and similar complication rates than those receiving TEA. As life expectancy increases, many TEA patients become too old to undergo surgery when the lifespan of their prostheses ends, resulting in many cases where patients have no choice but to bear the worn-out prosthesis for longer, i.e., up to 20 to 30 years. Therefore, rather than performing primary TEA right away just because the patient is elderly, if it is possible to perform osteosynthesis, ORIF can be a better choice for older patients with intra-articular distal humeral fractures.

\section{ORCID}

Jong Seok Baik https://orcid.org/0000-0002-1877-1837 Sung Hyun Lee https://orcid.org/0000-0001-6468-1456 Hyun Tak Kang https://orcid.org/0000-0001-8050-3246 Tae Hyun Seong Jeong Woo Kim https://orcid.org/0000-0001-9629-4856 https://orcid.org/0000-0002-0828-7179

\section{REFERENCES}

1. Robinson CM, Hill RM, Jacobs N, Dall G, Court-Brown CM. Adult distal humeral metaphyseal fractures: epidemiology and results of treatment. J Orthop Trauma 2003;17:38-47.

2. Zalavras CG, Vercillo MT, Jun BJ, Otarodifard K, Itamura JM, Lee TQ. Biomechanical evaluation of parallel versus orthogonal plate fixation of intra-articular distal humerus fractures. J Shoulder Elbow Surg 2011;20:12-20.

3. Shin SJ, Sohn HS, Do NH. A clinical comparison of two different 
double plating methods for intraarticular distal humerus fractures. J Shoulder Elbow Surg 2010;19:2-9.

4. Hackl M, Wegmann K, Bartsch V, Ries C, Burkhart KJ, Muller LP. Impingement of the posterolateral humeral plate: landmarks for prevention in double-plate osteosynthesis of distal humeral fractures. Obere Extremitat 2014;9:186-91.

5. Korner J, Lill H, Muller LP, et al. Distal humerus fractures in elderly patients: results after open reduction and internal fixation. Osteoporos Int 2005;16:S73-9.

6. McKee MD, Wilson TL, Winston L, Schemitsch EH, Richards RR. Functional outcome following surgical treatment of intra-articular distal humeral fractures through a posterior approach. J Bone Joint Surg Am 2000;82:1701-7.

7. McKee MD, Jupiter JB. Fractures of the distal humerus. In: Browner B, Jupiter J, Levine A, Trafton P, eds. Skeletal trauma. 3rd ed. Philadelphia, PA: Lippincott; 2002. p. 765-82.

8. O'Driscoll SW. Optimizing stability in distal humeral fracture fixation. J Shoulder Elbow Surg 2005;14 Suppl S:186S-194S.

9. Sodergard J, Sandelin J, Bostman O. Postoperative complications of distal humeral fractures. 27/96 adults followed up for 6 (2-10) years. Acta Orthop Scand 1992;63:85-9.

10. Korner J, Lill H, Muller LP, Rommens PM, Schneider E, Linke B. The LCP-concept in the operative treatment of distal humerus fractures: biological, biomechanical and surgical aspects. Injury 2003;34 Suppl 2:B20-30.

11. McKee MD, Pugh DM, Richards RR, Pedersen E, Jones C, Schemitsch EH. Effect of humeral condylar resection on strength and functional outcome after semiconstrained total elbow arthroplasty. J Bone Joint Surg Am 2003; 85:802-7.

12. Morrey BF, Adams RA, Bryan RS. Total replacement for post-traumatic arthritis of the elbow. J Bone Joint Surg Br 1991; 73:607-12.

13. Morrey BF, Adams RA. Semiconstrained elbow replacement for distal humeral nonunion. J Bone Joint Surg Br 1995;77:67-72.

14. Fracture and dislocation compendium: Orthopaedic Trauma Association Committee for Coding and Classification. J Orthop Trauma 1996;10 Suppl 1:v-ix. 1-154.
15. Armstrong AD, Yamaguchi K. Total elbow anthroplasty and distal humerus elbow fractures. Hand Clin 2004;20:475-83.

16. John H, Rosso R, Neff U, Bodoky A, Regazzoni P, Harder F. Operative treatment of distal humeral fractures in the elderly. J Bone Joint Surg Br 1994;76:793-6.

17. Jupiter JB, Neff U, Holzach P, Allgower M. Intercondylar fractures of the humerus. An operative approach. J Bone Joint Surg Am 1985;67:226-39.

18. Morrey BF, Chao E, Cooney W, et al. Indications in patient selection. In: Morrey BF, ed. Joint replacement arthroplasty. New York, NY: Churchill Livingstone; 1991. p. 275-91.

19. Gambirasio R, Riand N, Stern R, Hoffmeyer P. Total elbow arthroplasty as primary treatment for distal humeral fractures in elderly patients. J Bone Joint Surg Br 2001; 83:974-78.

20. Garcia JA, Mykula R, Stanley D. Complex fractures of the distal humerus in the elderly: the role of total elbow replacement as primary treatment. J Bone Joint Surg Br 2002;84:812-6.

21. Frankle MA, Herscovici D Jr, DiPasquale TG, Vasey MB, Sanders RW. A comparison of open reduction and internal fixation and primary total elbow arthroplasty in the treatment of intraarticular distal humerus fractures in women older than age 65. J Orthop Trauma 2003;17:473-80.

22. McKee MD, Veillette CJ, Hall JA, et al. A multicenter, prospective, randomized, controlled trial of open reduction--internal fixation versus total elbow arthroplasty for displaced intra-articular distal humeral fractures in elderly patients. J Shoulder Elbow Surg 2009;18:3-12.

23. Egol KA, Tsai P, Vazques O, Tejwani NC. Comparison of functional outcomes of total elbow arthroplasty vs plate fixation for distal humerus fractures in osteoporotic elbows. Am J Orthop (Belle Mead NJ) 2011;40:67-71.

24. Obremskey WT, Bhandari M, Dirschl DR, Shemitsch E. Internal fixation versus arthroplasty of comminuted fractures of the distal humerus. J Orthop Trauma 2003;17:463-5.

25. Savvidou OD, Zampeli F, Koutsouradis P, et al. Complications of open reduction and internal fixation of distal humerus fractures. EFORT Open Rev 2018;3:558-67. 